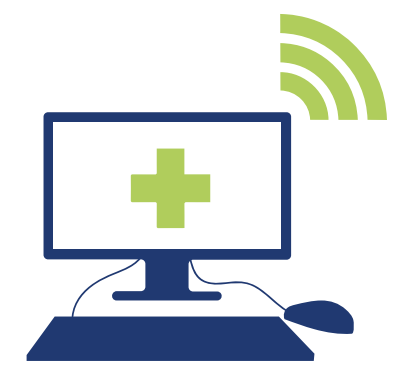

\title{
A TELESSAÚDE COMO FERRAMENTA NA EDUCAÇÃO CONTINUADA PARA O DIAGNÓSTICO PRECOCE DO CÂNCER INFANTOJUVENIL
}

TELEMEDICINE AS A TOOL IN CONTINUED EDUCATION FOR THE EARLY DIAGNOSIS OF

CHILD AND ADOLESCENT CANCER

\author{
Débora Rafaelly da Silva Vicente \\ Enfermeira Assistencial do Hospital Regional do Oeste - SC, Brasil. \\ Email: vicentedr068@gmail.com \\ Vera Lúcia Lins de Morais \\ Médica pediatra do Hospital Universitário Oswaldo Cruz -PE, Brasil. \\ Email : veramorais02@gmail.com
}

Magaly Bushatsky

Docente de enfermagem da Escola Pernambucana de Saúde, pela Universidade de Pernambuco- PE, Brasil.

Email: magab@hotlink.com

Débhora Ísis Barbosa e Silva

Especialista em Residência em Enfermagem em Pneumologia pelo Instituto de Medicina Integral Professor

Fernando Figueira - PE, Brasil

Enfermeira da Educação Permanente do Hospital Otávio de Freitas - PE, Brasil.

Email: debhora.silva@nutes.ufpe.br

Paula Rejane Beserra Diniz

Doutora em Neurologia pela Universidade de São Paulo - SP, Brasil

Membro permanente da Pós-Neuro da Universidade Federal de Pernambuco - PE, Brasil.

Email: paula.diniz@nutes.ufpe.br

Mariana Boulitreau Siqueira Campos Barros

Mestre em Saúde Coletiva (UFPE)/ Professora assistente do núcleo de Enfermagem do Centro Acadêmico de Vitória de Santo Antão/UFPE. - PE, Brasil.

Email: marianabscbarros@gmail.com

Natália Maria Penha Coutinho

Mestre em Saúde Coletiva (UFPE)/ Enfermeira teleconsultora do Núcleo de telessaúde do Hospital das

clínicas da UFPE - PE, Brasil.

Email: nataliampc@hotmail.com
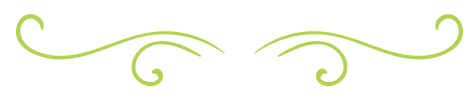

\section{RESUMO}

O câncer infantojuvenil vem se apresentando como a segunda causa de óbito na população entre 0 e 19 anos no Brasil, atrás apenas das causas externas. As tecnologias de informação e comunicação,

especificamente, a telessaúde, revelam-se como uma mola propulsora na triagem de suspeitas de neoplasias malignas, além de promover a integração da equipe da atenção primária à saúde e a do centro de 
referência, diminuindo distâncias, e promovendo educação continuada igualitária.

OBJETIVO: Associar os resultados do pré e pós-testes e descrever as teleconsultorias enviadas após a intervenção do projeto FIQUE ATENTO: PODE SER CÂNCER: A telessaúde como ferramenta para a suspeição precoce do câncer infantojuvenil, realizado com profissionais da atenção primária à saúde em Recife-Pernambuco.

MÉTODO: Trata-se de um estudo quase-experimental sem grupo controle, descritivo, com abordagem quantitativa. Realizado nos anos de 2015 e 2016, a partir da análise de dados secundários da Plataforma de Telessaúde HealthNET da RedeNutes, do Núcleo de Telessaúde da Universidade Federal de Pernambuco. Utilizou-se o software Epilnfo 7.2 como suporte estatístico para o cálculo das frequências absolutas e relativas, assim como para as medidas de associação. Adotou-se o p-valor menor que 0,05 para o cálculo de significância estatística. $O$ projeto foi aprovado sob o número do CAAE 50707515.7.0000.5208.

RESULTADOS: Os profissionais, após a intervenção, mostraram maior desenvolvimento com relação aos conhecimentos obtidos sobre epidemiologia $(p<0,001)$, e sinais e sintomas das neoplasias infantojuvenis $(p<0,001)$. Foram geradas oito teleconsultorias com tempo médio de resposta de 53,32 horas, em que 50\% (4) foram casos clínicos e tiveram seus encaminhamentos qualificados, e nenhum precisou ser regulado à unidade de referência.

CONCLUSÃO: A telessaúde mostra-se como uma ferramenta com potencial de educação permanente, contribuindo para o diagnóstico precoce do câncer infantojuvenil, aumentando as chances de cura e sobrevida.

Palavras-chave: Saúde da Criança; Saúde do Adolescente; Neoplasias; Telessaúde; Atenção primária à saúde.

ABSTRACT: The childhood's cancer is the second most common cause of death in the population between 0 and 19 years in Brazil, behind only of external causes. The information and communication technologies to health, such as telemedicine, they're a propulsive spring triaging suspected malignant neoplasms, as well as promoting the integration of the primary health care team and the center of reference, reducing distances and promoting education on an equal basis.

OBJECTIVE: To describe and to evaluate the results of pre and post-tests and teleconsultations sent after the intervention of the project BE AWARE: CAN BE CANCER! The telemedicine as a tool for the early suspicion of child and adolescent cancer, performed with primary health care professionals in Recife-Pernambuco.

METHOD: This is a cross-sectional, descriptive study with a quantitative approach, carried in the years 2015 and 2016, from the secondary data analysis of Telehealth platform HealthNET of RedeNUTES, Center for Telehealth at the Federal University of Pernambuco. The Epilnfo 7.2 software was used as statistical support for the calculation of absolute and relative frequencies, as well as for association measures. A p-value of less than 0.05 was used for the calculation of statistical significance. The project was approved under the number of CAAE 50707515.7.0000.5208.

RESULTS: After the intervention, the professionals showed greater development in relation to the knowledge obtained on epidemiology $(p<0,001)$ and early signs and symptoms of the child and adolescent neoplasm $(p<0,001)$. Eight teleconsultations were generated with an average response time of 53.32 hours, $50 \%$ (4) were clinical cases and and had their skilled forwards, none needed to be regulated to the reference unit.

CONCLUSION: Telemedicine presents a potential tool of permanent education, thus contributing to the early diagnosis of the children's cancer and, consequently, the chances of cure and survival. 
KEYWORDS: Child Health; Adolescent Health; Neoplasms; Telemedicine; Primary Health Care.

\section{INTRODUÇÃO}

No Brasil, as neoplasias apresentam-se como a segunda causa de óbito na população entre 0 e 19 anos, ficando atrás apenas das causas externas. O número de óbitos em 2014 foi de 2.724 crianças e adolescentes, e a estimativa para o biênio 2016/2017 é que ocorrerão cerca de 12.600 novos casos de câncer pediátrico em cada ano $(1,2)$.

Em relação às regiões geográficas do país supracitado, há grande discrepância com relação à taxa de mortalidade das neoplasias infantojuvenis, o Norte e o Nordeste apresentaram aumento destas taxas enquanto que nas regiões Sul, Sudeste e CentroOeste houve certa tendência ao declínio do número de óbitos por esta patologia. Tais resultados podem sugerir a existência de diferenças no acesso ao diagnóstico e tratamento da doença no país $(2,3)$.

As neoplasias na infância não são patologias preveníveis, pois, diferente de vários tipos de câncer de adultos, os fatores de risco relacionados ao estilo de vida não influenciam o risco de câncer de uma criança ou adolescente. Desta forma, a prevenção do câncer infantil ainda é um grande desafio, e a principal ferramenta para um melhor prognóstico está ligada ao diagnóstico precoce e encaminhamento imediato para tratamento, que vem possibilitar maiores taxas de cura. Cerca de $80 \%$ dos casos podem ser curados se detectados na fase inicial da patologia e tratados em centros especializados $(1,2,4)$.

Existem outras barreiras que acarretam o atraso no diagnóstico do câncer infantojuvenil, como: a sintomatologia e os sinais do câncer na infância são semelhantes a outras doenças comuns nessa faixa de idade; o despreparo dos profissionais em identificar precocemente a neoplasia; a dificuldade ao acesso dos serviços de saúde; um diálogo fragilizado entre os responsáveis, as crianças e os profissionais, que por vezes, refletem em longas trajetórias a serem percorridas para o reconhecimento do problema (5).

Para minimizar estes impasses, nos modelos alternativos de assistência à saúde, destaca-se a relevância da atuação da Atenção Primária à Saúde (APS), ou seja, das equipes de Estratégia de Saúde da Família (ESF), considerando ser esta a preferencial porta de entrada na rede de atenção à saúde, porque é nesse nível da assistência que acontece o primeiro contato, o acolhimento e a formação de vínculo da população com o sistema de saúde (6).

Entre os serviços oferecidos na ESF, a telessaúde disponibiliza a tele-educação e as teleconsultorias, que têm como finalidade promover uma educação permanente e a comunicação entre os profissionais da APS e os especialistas $(7,8)$.

A tele-educação, um dos serviços fornecidos, permite agir de forma colaborativa, promovendo equidade de conhecimentos, diminuição de distâncias e redução de custos. Faz a integração entre APS com áreas de excelência e ensino complementares em saúde e educação, permite o compartilhamento de conteúdos educacionais e assistenciais, promovendo uma educação continuada através de webconferência $(9,10)$.

Já a teleconsultoria pode ser delineada como uma consulta registrada e realizada entre profissionais e gestores da área de saúde, por meio de instrumentos de telecomunicação bidirecional, com o fim de esclarecer dúvidas sobre procedimentos clínicos, ações em saúde, ou questões relativas ao processo de trabalho. Pode ocorre de forma síncrona (em tempo real/online) ou assíncrona (mensagens offline) ${ }^{(7,11)}$.

Para o câncer infantojuvenil, a telessaúde revela-se uma mola propulsora, promovendo uma comunicação entre os profissionais da APS e os oncologistas pediátricos por meio dessas tecnologias de informação e comunicação (TICs), esclarecendo dúvidas sobre o diagnóstico 
precoce, procedimentos e envio de casos clínicos suspeitos.

Neste cenário, os objetivos do presente estudo foram associar os resultados do pré e pós-testes e descrever as teleconsultorias enviadas após a intervenção do projeto FIQUE ATENTO: PODE SER CÂNCER: A telessaúde como ferramenta para a suspeição precoce do câncer infantojuvenil, realizado com profissionais da atenção primária à saúde em Recife-Pernambuco.

\section{MATERIAIS E MÉTODOS}

Trata-se de um estudo quase-experimental sem grupo controle, descritivo com abordagem quantitativa, realizado na cidade do Recife, capital de Pernambuco, nos Distritos Sanitários (DS) II e IV. A delimitação desses distritos sanitários da cidade supracitada justifica-se pelo primeiro ser campo de prática da Universidade de Pernambuco, parceira no projeto, e pelo DS IV possuir, conforme dados enviados pela Secretária Municipal de Saúde da cidade do Recife-PE, o maior percentual de mortalidade por câncer infantil (20\%) entre os oitos distritos que compõem o local do estudo.

A pesquisa utilizou dados gerados durante o período de novembro de 2015 a dezembro de 2016. A população do estudo foi composta pelos os profissionais da atenção primária à saúde dos DS II e IV, num total de 982 pessoas, com diferentes categorias e especialidades, tais como agente comunitário de saúde da família, assistentes sociais, equipe da recepção da Unidade Básica de Saúde (UBS), fisioterapeutas, fonoaudiólogos, médicos, nutricionistas, psicólogos, técnicos e auxiliares de enfermagem, enfermeiros, técnicos e auxiliares de saúde bucal e cirurgiões dentistas.

Calculou-se, com suporte do Epilnfo 7.2, uma amostra mínima necessária de 276 pessoas, com a frequência esperada de $50 \%$ e nível de significância de 5\%. Foram excluídos da amostra 262 profissionais que só realizaram o pré-teste, 20 que só realizaram o pós-teste, 187 que tiveram menos de $75 \%$ de presença nas aulas, e 23 que se recusaram a assinar o Termo de consentimento e livre esclarecimento (TCLE), restando assim uma amostra de inclusão de 490 profissionais.

Desta forma, na Tabela 1 foram elucidadas as categorias profissionais entre os 490 trabalhadores da atenção primária à saúde dos DS II e IV, que compõe a amostra do estudo em questão, da cidade do Recife-PE, participantes do projeto. Houve a predominância de agentes comunitários de saúde com 48,6\% (238), seguida por enfermeiros $7,8 \%$ (38) e médicos com 5,1\% (25).

Tabela 1 - Caracterização dos participantes do estudo. Recife- PE, 2015 e 2016.

\begin{tabular}{|c|c|c|}
\hline CATEGORIA PROFISSIONAL & $\mathbf{N}$ & $\%$ \\
\hline Agentes comunitários de saúde & 238 & 48,6 \\
\hline Enfermeiros & 38 & 7,8 \\
\hline Médicos & 25 & 5,1 \\
\hline Técnicos e/ou auxiliares de enfermagem & 22 & 4,5 \\
\hline $\begin{array}{l}\text { Equipe de saúde bucal: cirurgião dentista, auxiliar de saúde bucal e técnico em } \\
\text { saúde bucal. }\end{array}$ & 53 & 10,8 \\
\hline $\begin{array}{l}\text { Equipe do NASF: assistente social, fisioterapeuta, fonoaudiólogo, nutricionista, } \\
\qquad \text { psicólogo e terapeuta ocupacional. }\end{array}$ & 9 & 1,8 \\
\hline
\end{tabular}


A fim de alcançar o objetivo pressuposto na intervenção, o projeto FIQUE ATENTO: PODE SER CÂNCER atuou em três eixos estruturantes:

\section{I - Sensibilização}

Antes de dar início à ação propriamente dita, foi realizada uma sensibilização e divulgação para os profissionais da atenção básica, nas próprias unidades de saúde, junto aos profissionais, e também através da mídia, com reportagens em jornais e televisão, como também em sites e redes sociais, em relação à importância do diagnóstico precoce do câncer infantojuvenil.

\section{II - Tele-educação: a realização do Curso "FIQUE ATENTO: PODE SER CÂNCER!"}

O curso foi transmitido para 42 pontos de transmissão distribuídos no próprio território de abrangência dos DS II e DS IV, com frequência semanal e duração de cinco semanas, resultando na carga horária total de 10 horas.

As aulas foram ministradas e transmitidas de forma síncrona, via webconferência pelo Núcleo de Telessaúde (NUTES) da Universidade de Pernambuco (UPE) com o suporte do NUTES da Universidade Federal de Pernambuco (UFPE) e sua transmissão foi realizada através da Plataforma INDU programada pelo NUTES da UFPE.

As aulas foram divididas nos seguintes temas: 1) Política Nacional para a prevenção e controle do câncer na rede de atenção à saúde das pessoas com doenças crônicas;

2) Epidemiologia do câncer infantojuvenil; 3) Os sinais e sintomas de suspeição precoce do câncer infantojuvenil; 4) A ESF e o cuidado da criança e do adolescente com câncer, a organização do sistema de referência e contrarreferência para a assistência à criança e ao adolescente com câncer; e 5) $\bigcirc$ uso da Plataforma de Telessaúde HealthNET.

\section{III - Teleconsultorias: O uso da plata- forma HealthNET e a construção da rede de referência por telessaúde}

A teleconsultoria pela Plataforma HealthNET da RedeNUTES foi apresentada como aliada para a suspeição precoce do câncer infantojuvenil, bem como para responder dúvidas de questões clínicas. Este recurso também possibilitou a construção da rede de encaminhamento, quando se formou uma equipe de teleconsultores composta por especialistas em oncologia pediátrica, composta por profissionais do Hospital Universitário Osvaldo Cruz, referência estadual nos cuidados de crianças e adolescentes com câncer, que, juntamente com os coordenadores do projeto, formaram uma rede de encaminhamento de casos suspeitos enviados pela teleconsultoria, assim como a garantia da contrarreferência.

A plataforma HealthNET está disponível em aplicativo móvel, e a mesma foi desenvolvida pelo Núcleo de Telessaúde - NUTES do Hospital das Clinicas da UFPE para auxiliar profissionais de saúde em questões de processos de trabalho e diagnóstico a distância. Essa tecnologia já é utilizada na oferta dos serviços de telessaúde da Rede de NUTES de Pernambuco - RedeNUTES.

Para descrever o conhecimento dos profissionais antes e após o curso, foram aplicados pré-testes (no primeiro dia do 
curso) e pós-testes (no último dia do curso) através de um questionário estruturado, impresso em folha de papel e entregue aos participantes, com as mesmas perguntas nos dois momentos. $O$ instrumento foi elaborado pelos palestrantes, contendo questões sobre epidemiologia, sinais e sintomas e como proceder em caso de suspeição, com o fim de averiguar o nível de conhecimento dos participantes, e foram exploradas questões sobre a epidemiologia do câncer infantojuvenil: raridade do câncer infantojuvenil, a posição destas neoplasias no quadro de morbimortalidade, e os sinais e sintomas precoces das leucemias, linfomas e tumor do sistema nervoso central.

Utilizou-se os dados primários, resultados dos pré e pós-testes realizados após a transmissão por webconferência do curso FIQUE ATENTO: PODE SER CÂNCER, sobre como os profissionais da atenção primária podem suspeitar precocemente de um câncer infantojuvenil.

Estes foram analisados por medidas absolutas e relativas e medidas de hipótese que foram calculados pelo teste de Quiquadrado, através de uma análise bivariada, tendo como variáveis independentes os acertos e os erros no pré-teste, somando-se aos desacertos os que não responderam. E como variáveis dependentes as mesmas alternativas do pós-teste. Adotou-se um nível de significância de 5\% e, para análise, utilizou-se o Excel 2007 e o software Epilnfo versão 7.2.

Para as teleconsultorias, a análise dos dados ocorreu por estatística descritiva, realizada por medidas absolutas e relativas assim como de tendência central e de dispersão, com o suporte do software Epilnfo versão 7.2.

$O$ perfil das teleconsultorias foi traçado através do detalhamento das mesmas, que foram geradas após a intervenção do projeto de extensão Fique atento! Pode ser câncer, sobre as variáveis: 1) Tempo de resposta; 2) Ocupação do Solicitante; 3) Ocupação do teleconsultor; 4) Especialidade do teleconsultor; 5) Natureza da teleconsultoria.

Foi adotado como critério de inclusão as teleconsultorias geradas com todos os dados devidamente preenchidos, cuja natureza seja caso clínico, questão clínica ou questão de processo de trabalho.

O projeto foi cadastrado na Plataforma Brasil e encaminhado ao Comitê de Ética em Pesquisa do Centro de Ciências da Saúde da UFPE, sendo aprovado sob o número de protocolo CAAE 50707515.7.0000.5208. A pesquisa respeitou a Resolução 466/2012 do Conselho Nacional da Saúde com relação à pesquisa envolvendo seres humanos, e foi mantido o anonimato e consentimento dos participantes da pesquisa através da assinatura do TCLE.

\section{RESULTADOS E DISCUSSÃO}

Desta forma, os resultados obtidos através das respostas dos pré e pós-testes foram demonstrados nas tabelas seguintes.

$\mathrm{Na}$ Tabela 2 verifica-se que o conhecimento sobre a epidemiologia do câncer infantojuvenil, pelos profissionais da atenção primária dos DS II e IV em Recife, foi aprimorado no pós-teste, com exceção sobre a raridade da neoplasia em crianças e adolescentes. Observa-se que o teste de associação sobre a posição das neoplasias no quadro de mortalidade e o câncer mais incidente em Pernambuco foi significativo com p-valor menor que 0,001 e 0,026, respectivamente, indicando que a intervenção do curso FIQUE ATENTO: PODE SER CÂNCER foi fator determinante para a mudança relacionada ao conhecimento epidemiológico do câncer infantojuvenil entre os profissionais participantes. 
Tabela 2 - Análise dos Pré e Pós-teste com relação à epidemiologia do câncer infantojuvenil. Recife- PE, 2015 e 2016.

\begin{tabular}{|c|c|c|c|c|c|}
\hline \multicolumn{6}{|c|}{ Pergunta: O câncer é uma patologia comum na criança? } \\
\hline & \multicolumn{2}{|c|}{ Pré-teste } & \multicolumn{2}{|c|}{ Pós-teste } & \multirow{2}{*}{ p-valor* } \\
\hline & $\mathrm{N}$ & $\%$ & $\mathrm{~N}$ & $\%$ & \\
\hline Verdadeiro & 236 & 48,2 & 265 & 54 & \\
\hline Falso & 254 & 51,8 & 225 & 46 & 0,064 \\
\hline Total & 490 & 100 & 490 & 100 & \\
\hline \multicolumn{6}{|c|}{ Pergunta: Qual a posição das neoplasias infantojuvenis (de 1 a 19 anos) no quadro de mortalidade nacional? } \\
\hline & \multicolumn{2}{|c|}{ Pré-teste } & \multicolumn{2}{|c|}{ Pós-teste } & \multirow[t]{2}{*}{ p-valor* } \\
\hline & $\mathrm{N}$ & $\%$ & $\mathrm{~N}$ & $\%$ & \\
\hline Primeiro e Segundo** & 264 & 53,9 & 390 & 79,6 & \multirow{3}{*}{$<0,001$} \\
\hline Demais posições & 226 & 46,1 & 100 & 20,4 & \\
\hline Total & 490 & 100 & 490 & 100 & \\
\hline \multicolumn{6}{|c|}{ Pergunta: Qual o tipo de neoplasia infantil mais frequente em Pernambuco? } \\
\hline & \multicolumn{2}{|c|}{ Pré-teste } & \multicolumn{2}{|c|}{ Pós-teste } & \multirow[b]{2}{*}{ p-valor* } \\
\hline & $\mathrm{N}$ & $\%$ & $\mathrm{~N}$ & $\%$ & \\
\hline Leucemias & 395 & 80,6 & 421 & 85,9 & \multirow{3}{*}{0,026} \\
\hline Demais neoplasias & 95 & 19,4 & 69 & 14,1 & \\
\hline Total & 490 & 100 & 490 & 100 & \\
\hline
\end{tabular}

Fonte: Elaboração própria com dados secundários da RedeNUTES, 2017.

* Teste de Quiquadrado Uncorrected, IC: 95\%.

** Foram consideradas as alternativas "Primeira" e "Segunda" como corretas.

O câncer infantojuvenil é considerado raro quando comparado à incidência em adultos, correspondendo a uma pequena porcentagem definida como sendo de 1 a $3 \%$ de todos os novos casos de neoplasias malignas no mundo. Contudo, as neoplasias vêm se apresentando como a primeira causa de óbito por doença e a segunda causa de óbito na população entre 0 e 19 anos, atrás apenas das causas externas, como acidentes, por exemplo $(2,12-14)$. 
Dentre o conjunto de neoplasias que mais atinge esse grupo, as leucemias são as mais frequentes, sua taxa média de incidência ajustada por idade, para o período de 2009 a 2013, foi de 14,94 por milhão de crianças e adolescentes, seguida dos tumores do sistema nervoso central com taxa de 10,26 por milhão, e dos linfomas não Hodgkin (LNH) com 2,70 por milhão e linfomas de Hodgkin, 0,56 por milhão ${ }^{(13,15)}$. Por esta razão perguntou-se se os profissionais saberiam identificar os sinais e sintomas clínicos dessas neoplasias, e os resultados foram descritos na Tabela 2.
Verifica-se que o conhecimento sobre os sinais e sintomas dos tipos de câncer infantojuvenil, pelos profissionais da atenção primária dos DS II e IV em Recife, Pernambuco, foi aprimorado no pós-teste. Ainda, observa-se que o teste de independência foi significativo ( $p$-valor $<0,001$ ), indicando que a intervenção do curso FIQUE ATENTO: PODE SER CÂNCER foi fator determinante para a mudança sobre o conhecimento dos sinais e sintomas das leucemias, tumores do sistema nervoso central e linfomas no público infantojuvenil, entre os profissionais participantes.

Tabela 3 - Distribuição das respostas do Pré e Pós-teste segundo os sinais e sintomas precoce do câncer infantojuvenil. Recife- PE, 2015 e 2016

Sinais e Sintomas: Dor de cabeça, náuseas/vômitos, visão turva ou dupla, tontura e dificuldade para caminhar ou manipular objetos.

\begin{tabular}{|c|c|c|c|c|c|}
\hline & \multicolumn{2}{|c|}{ Pré-teste } & \multicolumn{2}{|c|}{ Pós-teste } & \multirow{2}{*}{ p-valor* } \\
\hline & $N$ & $\%$ & $\mathrm{~N}$ & $\%$ & \\
\hline Tumor SNC & 325 & 66,33 & 379 & 77,35 & \multirow{3}{*}{$<0,001$} \\
\hline Outras Neoplasias & 165 & 33,67 & 111 & 22,65 & \\
\hline Total & 490 & 100 & 490 & 100 & \\
\hline
\end{tabular}

Sinais e Sintomas: Palidez, fadiga, febre, dor óssea, hepatoesplenomegalia.

\begin{tabular}{|c|c|c|c|c|c|}
\hline & \multicolumn{2}{|c|}{ Pré-teste } & \multicolumn{2}{|c|}{ Pós-teste } & \multirow[t]{2}{*}{ p-valor* } \\
\hline & $\mathrm{N}$ & $\%$ & $\mathrm{~N}$ & $\%$ & \\
\hline Leucemias & 334 & 68,16 & 398 & 81,22 & \multirow{3}{*}{$<0,001$} \\
\hline Outras Neoplasias & 156 & 31,84 & 92 & 18,78 & \\
\hline Total & 490 & 100 & 490 & 100 & \\
\hline
\end{tabular}

Sinais e Sintomas: Adenomegalia, febre sudorese noturna, perda de peso.

\begin{tabular}{|l|l|l|l|}
\hline \multicolumn{2}{|c|}{ Pré-teste } & \multicolumn{2}{c|}{ Pós-teste } \\
\hline \\
$N$
\end{tabular}




\begin{tabular}{|c|c|c|c|c|c|}
\hline Linfomas & 292 & 59,59 & 354 & 72,25 & \\
\hline Outras Neoplasias & 198 & 40,41 & 136 & 27,75 & $<0,001$ \\
\hline Total & 490 & 100 & 490 & 100 & \\
\hline
\end{tabular}

Fonte: Elaboração própria com dados secundários da RedeNUTES, 2017.

* Teste de Quiquadrado Uncorrected, IC: 95\%.

De fato, nas leucemias, a criança tende a se tornar mais sujeita a infecções, podendo apresentar palidez, anemias, sangramento, perda de peso sem motivo aparente, gânglios linfáticos inchados, hepatoesplenomegalia e sentir dores ósseas, corroborando com os resultados apresentados na Tabela 3. Essa sintomatologia se dá decorrente do acúmulo de células defeituosas na medula óssea, prejudicando ou impedindo a produção dos glóbulos vermelhos, dos glóbulos brancos e das plaquetas $(2,16)$.

Já os tumores do sistema nervoso central, podem provocar edema cerebral ou bloqueio do fluxo do líquido cefalorraquidiano (LCR), e o aumento da pressão em qualquer um desses pontos pode causas sinais em sintomas, concordante com o exposto na Tabela 3, como cefaleia, náuseas, visão turva ou dupla, problemas com o equilíbrio, alterações comportamentais, ou até mesmo convulsões e sonolência (17).

Enquanto que os linfomas podem surgir em qualquer região do corpo e os sintomas estão diretamente ligados a sua localização, por exemplo, se a doença ocorre na região do tórax, tosse, falta de ar e dor torácica podem se manifestar. Outros sinais de alerta que podem se manifestar são: aumento dos gânglios linfáticos sem dor; febre; fadiga; sudorese noturna; perda de peso sem causa aparente; prurido e esplenomegalia, corroborando com os resultados apresentados na Tabela $3(16,18)$.

Com relação às teleconsultorias geradas, pôde-se observar que nos dois anos de atuação do FIQUE ATENTO! PODE SER CÂNCER, foram enviadas oito teleconsultorias, assíncronas, pela plataforma HealthNET, com média de tempo para resposta de 52,92 horas, e desvio padrão de +/- 50,18 horas, com menor amplitude de tempo de resposta de 21,83 horas e maior de 642,28 horas.

Destas, $50 \%$ (4) foram casos clínicos, $37,5 \%$ (3) de questões clínicas e 12,5\% (1) de questão de processo de trabalho. A maior parte das teleconsultorias enviadas após a intervenção do curso, que correspondem a $62,5 \%$ (5), foram enviadas por enfermeiros e respondidas respectivamente por médicos oncologistas pediátricos, ou médicos da família e da comunidade.

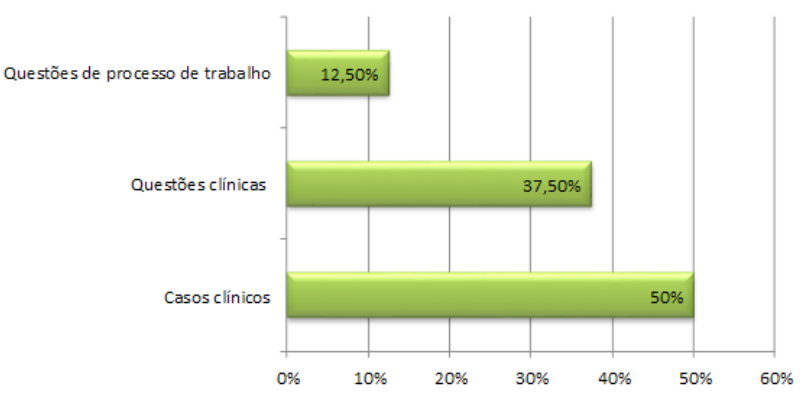

Figura 1 - Gráfico de distribuição da natureza das teleconsultorias, 2015.

Fonte: Elaboração própria com dados secundários da RedeNUTES, 2017.

Nenhum caso clínico enviado precisou de encaminhamento para unidade de referência, todos os casos suspeitos foram qualificados com suporte dos teleconsultores, e evitados os deslocamentos desnecessários para a atenção terciária.

O tempo médio de resposta condiz com - que a Portaria $N^{\circ} 2.546$ de outubro de 2011 estabelece, ou seja, está dentro do prazo máximo de 72 (setenta e duas) horas, contanto a partir do recebimento da mesma 
As Teleconsultorias devem ser respondidas pelos Núcleos Técnico-Científicos, pelo profissional de referência daquela demanda, baseado nas melhores e atualizadas evidências clínicas e científicas disponíveis, sendo que a resposta deve ressaltar o conhecimento que subsidie desenvoltura do problema e que venha a contribuir com a educação permanente dos profissionais envolvidos, visando cada vez mais a ampliação de sua capacidade e autonomia na resolução futura de casos semelhantes $(7,19)$.

O núcleo de Telessaúde do Rio Grande do Norte, em 2015, realizou um estudo usando dados do período de abril à dezembro de 2014, no qual o levantamento das teleconsultorias revelou que diferente dos achados do atual estudo, a maior parte das teleconsultorias, correspondendo a 45\% (21), foram geradas por agentes comunitários de saúde e 93,6\% (44) de natureza questão clínica (11).

Quando identificada a necessidade de encaminhamento, o Telessaúde Brasil Redes deve promover a articulação à regulação da oferta de serviços, em parceria com a gestão de saúde, de forma compartilhada e articulada com os pontos de atenção da rede ${ }^{(7)}$.

\section{CONCLUSÃO}

Os profissionais da atenção primária à saúde dos DS II e IV da cidade do Recife, Pernambuco, mostraram pelas respostas dos pré e pós-testes, que a intervenção do projeto FIQUE ATENTO! PODE SER CÂNCER colaborou para o aprimoramento dos conhecimentos sobre a epidemiologia das neoplasias no público infantojuvenil e também sobre os sinais e sintomas das neoplasias mais recorrentes a este grupo. Além disso, o curso contribuiu para empoderar e divulgar a esses profissionais o uso da teleconsultoria, aproximando os níveis de atenção à saúde.

Considerando a significância dos dados relacionados à evolução dos conhecimentos da população amostral, após a intervenção, torna-se importante a continuidade da educação permanente dos profissionais de saúde a partir da expansão e fortalecimento da tele-educação, investindo mais em conectividade dos pontos de atenção à saúde, na capacitação dos profissionais por ambientes virtuais, e no uso de ferramentas tecnológicas que fortaleçam a educação continuada.

Em relação às teleconsultorias, observa-se a importância de implementar uma linha de cuidados dos casos suspeitos de câncer infantojuvenil no estado de Pernambuco, e fortalecer a referência e contrarreferência pela telessaúde.

As limitações do estudo giraram em torno de possíveis vieses de confundimento sobre o conhecimento dos profissionais acerca da epidemiologia do câncer na criança e no adolescente e sua posição no primeiro ou segundo lugar no ranking nacional de mortalidade, assim como esta ser uma patologia comum na infância, além dos vieses de informação por haver várias categorias participantes com níveis de instrução diferentes.

Durante a pesquisa constatou-se a ausência de estudos com objetos semelhantes, que utilizem a telessaúde como ferramenta para diagnósticos de neoplasias infantojuvenis ou mesmo sobre patologias da criança e do adolescente. A escassez dessas publicações caracteriza o pioneirismo do estudo, elucidando e fortalecendo a necessidade de pesquisas nessa área.

Expandir esse estudo a outros distritos sanitários e a outras regiões torna-se necessário com fins de aumentar o alcance da suspeição precoce dessa patologia, como também o desenvolvimento de mais estudos envolvendo o uso das TICs, em especial, a telessaúde. 


\section{REFERÊNCIAS BIBLIOGRÁFICAS}

INCA. Particularidades do Câncer Infantil. - INCA [ Internet ], 2015. Acessado em 30 de julho de 2016. p. 8-9. Disponível em: http://www. inca.gov.br/conteudo_view.asp?id=343.

INCA. Tipos e incidência do câncer infantil. - INCA [Internet],2017. Acessado em 28 de maio de 2017. Disponível em: http://www2.inca. gov.br/wps/wcm/connect/tiposdecancer/site/home/infantil.

Santos, D.; Echenique, I.; Texeira, L. R.. Mortalidade por câncer infantojuvenil: entenda a queda. Rede Câncer [ Internet]. 2010;40-1. Acessado em 18 fevereiro de 2017. Disponivel em: http://www2.inca. gov.br/wps/wcm/connect/bf9362004140c0bfadadade8765d5801/13_ artigo.pdf?MOD=AJPERES.

American Cancer Society. Can Childhood Cancers Be Prevented? [Internet]. Cancer in children. 2016. Acessado em 28 de maio de 2017. Disponível em: https://www.cancer.org/cancer/cancer-in-children/ preventing-childhood-cancers.html.

Coeli, M.; Viana, C.; Monteiro, A.I. Team's performance in primary health care on early identification of children's cancer-Revista Brasileita de Pesquisa: Cuidado é fundamental, Online. 2012;4(3):2692-701. Acessado em 20 de dezembro de 2016. Disponivel em: http://seer.unirio.br/index.php/cuidadofundamental/article/viewFile/1863/pdf_609.

Rodrigues, L.B.B.;Silva,P.C.dosS.;Peruhype, R.C.;Palha, P.F.;Popolin, M.P.; Crispim, J. de A.; et al. A atenção primaria à saude na coordenação das redes de atenção: Uma revisão integrativa. Ciências e Saúde Coletiva [Internet]. 2014;19(2):343-52. Acessado em 14 de janeiro de 2017. Disponível em: http://www.scielosp.org/scielo.php?script=sci_ arttext\&pid=S1413-81232014000200343\&lng=pt\&nrm=iso\&tlng=pt.

BRASIL. Ministério da Saúde. Gabinete do Ministro. Portaria Nº 2.546, de 27 de outubro de 2011.Redefine e amplia o Programa Telessaúde Brasil, que passa a ser denominado Programa Nacional Telessaúde Brasil Redes (Telessaúde Brasil Redes). Diário Oficial da União. Inciso II do parágrafo único do art. 87 da Constituição.

Brasil. Ministério da Saúde. Comissão Nacional de Ética em Pesquisa. Conselho Nacional de Saúde. Resulução N 466, de 12 de dezembro de 2012; 1:12. Acessado em 16 de março de 2018. Disponivel em: http://conselho.saude.gov.br/resolucoes/2012/reso466.pdf.

Batista, V.; Araújo, B. G.; Valentim, R. A. M.; Silva, J. L. R.; Morais, I. R. D. Uma abordagem jurídica sobre o projeto telessaúde. - Revista 
Brasileira de Inovação Tecnológica em saúde, online. - 2014;33-50. Acessado em 20 de janeiro de 2017. Disponível em: https://periodicos. ufrn.br/reb/article/view/5603.

Souza, C.; Pimenta, D. Videoconferência e webconferência na EAD, análise dos usos e perspectivas de aplicação. XI Congresso Brasileiro de ensino superior à distância. Florianópolis, SC, UNIREDE, 2014;5-8.

Lucena, B.E.B.; Diniz Junior, J.; Diniz, R. V. Z. Telessaúde como ferramenta de apoio à atenção primária à saúde: um olhar sobre as teleconsultorias em cardiologia. Rev Bras Inovação Tecnológica em Saúde. 2013;38-47. Acessado em 20 de janeiro de 2017. Disponivel em: https://periodicos.ufrn.br/reb/article/view/6998.

Mutti, C.F.; Paula, C.C.; Souto, M.D. Assistência à Saúde da Criança com Câncer na Produção Científica Brasileira Health Care of Children With Cancer in the Brazilian Scientific Literature. 2010. 2010;56(1):71-83.

Curvo, H.R.M.; Pignati, W.A.; Pignatti, M.G. Morbimortalidade por câncer infantojuvenil associada ao uso agrícola de agrotóxicos no Estado de Mato Grosso, Brasil. Caderno de Saúde Coletiva [Internet]. 2013;21(1):10-7. Acessado em novembro de 2016. Disponivel em: http://www.scielo.br/scielo.php?script=sci_arttext\&pi$d=S 1414-462 X 2013000100003 \&$ lng $=p t \& n r m=i s o \& t \mid n g=e n$.

Friestino, J. K. O.; Mendonça, D.; Oliveira, P.; Oliveira, C. M.; Ferraz, R. O.; Moreira Filho, D. C. Estatística Espacial Aplicada aos Estudos de Incidência de Câncer - Revista Eletrônica TEMPUS, Actas de Saúde Coletiva, Universidade de Brasília- Núcleo de Estudos de Saúde Pública. 2016;10(2):103-17. Acessado em 18 de fevereiro de 2017. Disponivel em: http://www.tempus.unb.br/index.php/tempus/article/ view/1772

INCA. Incidência, mortalidade e morbidade hospitalar por câncer em crianças, adolescentes e adultos jivens no Brasil: Rio de Janeiro; 2016. 414 p. Acessado em 22 de janeiro de 2016. Disponivel em : http:// www1.inca.gov.br/wcm/incidencia/2016/index.asp.

INCA. Tipos de câncer infantojuvenil [Internet]. 2016. Acessado em 30 de junho de 2016. Disponivel em: http://www2.inca.gov.br/wps/ wcm/connect/tiposdecancer/site/home/infantil

Instituto Oncoguia. Sinais e Sintomas dos Tumores Cerebrais / Sistema Nervoso Central em Crianças - Instituto Oncoguia [Internet]. 2017. Acessado em 28 de maio de 2017. Disponível em: http://www. oncoguia.org.br/conteudo/sinais-e-sintomas-dos-tumores-cerebrais---sistema-nervoso-central-em-criancas/4112/596/. 
ABRALE. LINFOMA EM CRIANÇAS - ABRALE [Internet]. Câncer Infantil. 2016. Acessado em 28 de maio de 2017.p. 12. Disponível em : http://www.abrale.org.br/linfoma-infantil/lnh

BRASIL. Ministério da Saúde. Telessaúde Brasil Redes [Internet]. Brasília; 2012. 73 p. Acessado em 14 de novembro de 2016. Disponível em: http://189.28.128.100/dab/docs/portaldab/publicacoes/manual_ telessaude.pdf. 